\title{
VARIATION DE LA CONSOMMATION SPONTANÉE D'ÉNERGIE DU POUSSIN EN FONCTION DE LA NATURE DES MATIÈRES AZOTÉES DU RÉGIME
}

\author{
C. CALET, C. JOUANDET et T. BARATOU \\ Station de Recherches avicoles, \\ Centre national de Recherches zootechniques, Jouy-en-Josas.
}

\section{SOIMIMAIRE}

On peut modifier l'efficacité des matières azotées du régime en distribuant simultanément, dans deux mangeoires séparées, les protéines et le reste de la ration, ou en fournissant ces deux éléments dans un même mélange. Alors que le gain de poids demeure sensiblement le même dans les deux cas lorsqu'il s'agit de l'arachide, la libre consommation des calories permet une augmentation de la croissance des animaux qui reçoivent le poisson. Pour une même quantité de matières azotées totales ingérées, la consommation spontanée d'énergie augmente de $30 \mathrm{p}$. Ioo lorsque l'on remplace l'arachide par le poisson. Il en résulte que le rapport optimum entre les calories et les protéines du régime dépend de la nature des matières azotées qu'il renferme.

Bien des hypothèses ont été proposées pour expliquer comment l'animal règle son appétit. Pour les uns (MAYER, I952, HARPER, KuMTA, I959), la composition du sang en glucose ou en acides aminés déterminerait le niveau des ingestats. Pour BroBECK, I948, PASSMORE et RITCHIE, I957, la production de chaleur consécutive à l'acte alimentaire représenterait une part importante du mécanisme de la satiété. HILL, et DANSKY, I954, montrent que le poussin consomme autant d'aliment compleł et équilibré qu'il est nécessaire pour couvrir son besoin énergétique. Ils constatent que la quantité de calories consommées par des animaux de même poids est constante et proportionnelle à leur poids vif élevé à la puissance 0,76 . Dans un autre ordre d'idées, LEVEILIE et FISHER, I958, mesurant les besoins en acides aminés du coq adulte, attirent l'attention sur le fait que le besoin énergétique augmente de 20 p. Ioo lorsque l'on remplace les acides aminés du protide, par des acides aminés libres.

Dans ces conditions, nous nous sommes demandés dans quelle mesure la nature de la source azotée modifiait la consommation de principes énergétiques de l'animal. Pour y parvenir, nous avons mesuré la consommation spontanée de calories métabolisables que le poussin ingère lorsqu'on distribue séparément les éléments azotés en quantité limitée et le reste de la ration. 


\section{MATÉRIEL ET MÉTHODES}

60 poussins issus du croisement Rhode et Wyandotte sont élevés en batterie. A l'âge de 3 semaines ils sont répartis en lots homogènes d'après leur poids et leur gain de poids pendant la période préexpérimentale, selon la méthode de Mac KITTRICK, 1947. Chacun des régimes est distribué à trois lots différents, de manière à avoir trois répétitions du même traitement. Pendant I 8 jours les poussins reçoivent la même quantité de matière azotée totale qui leur est fournie, soit sous forme de tourteau d'arachide, soit sous forme de farine de poisson. De plus, on distribue ces aliments, soit en les mélangeant au reste de la ration constituée par un régime sans azote, soit isolément. Dans le premier cas le mélange qui titre $200 \mathrm{~g}$ de protides et 3500 calories métabolisables par kilo, est distribué chaque jour en quantité limitée, à raison de $25,5 \mathrm{~g}$ par poussin. Dans le second cas, on apporte quotidiennement à chaque poussin 5, I $\mathrm{g}$ de la source azotée considérée dans une mangeoire, et dans une autre mangeoire le régime sans azote qui est fourni à volonté. L'animal a accès aux deux mangeoires simultanément. On mesure chaque jour la quantité ingérée du régime sans azote, de façon à apprécier la consommation spontanée d'énergie de l'animal. Le régime sans azote est un régime purifié qui renferme 72 p. roo d'hydrates de carbone, I 3 p. roo d'huile de maïs, 9 p. soo de complément minéral et $3 \mathrm{p}$. Ioo de complément vitaminique. Ces compléments sont établis de manière à procurer au poussin tous les éléments minéraux et vitaminiques dont il a besoin, selon les normes actuellement connues.

\section{RÉSULTATS}

L'influence du mode de distribution de l'aliment sur la valeur alimentaire du tourteau d'arachide figure dans le tablean I.

TABLEAU I

Valeur alimentaire du tourteau d'arachide selon le mode de rationnement.

\begin{tabular}{|c|c|c|}
\hline Mode de distribution des protides de la ration & $\begin{array}{c}\text { En mélange } \\
\text { Taux protéique } \\
\text { du régime } 20 \text { p. cent }\end{array}$ & Séparément \\
\hline 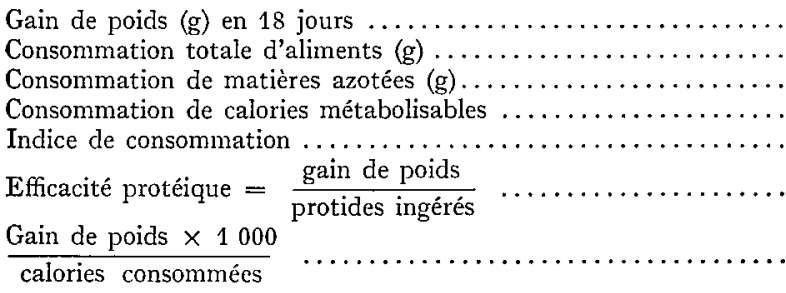 & $\begin{array}{r}114,4 \\
458,0 \\
92,6 \\
1628,0 \\
4,0 \\
\\
1,24\end{array}$ & $\begin{array}{r}110,2 \\
428,0 \\
92,4 \\
1420,0 \\
3,9 \\
\\
1,20\end{array}$ \\
\hline
\end{tabular}

Quel que soit le mode de rationnement, la valeur alimentaire de l'arachide demeure constante : même gain de poids, même indice de consommation, même efficacité protéique. On remarque seulement que les poussins ingèrent moins d'aliments totaux et moins de calories métabolisables pour faire le même gain de poids lorsqu'ils disposent des éléments énergétiques à volonté, que lotsqu'on leur impose de les consommer en mélange. Il en résulte que l'efficacité des calories dans ce dernier cas est diminuée.

Lorsque l'on s'adresse aux protides du poisson les résultats sont quelque peu différents comme le montre le tableau 2. 
TABLEAU 2

Influence du mode de distribution de l'aliment sur la valeur alimentaire de la farine de poisson.

\begin{tabular}{|c|c|c|}
\hline Mode de distribution des protides de la ration & $\begin{array}{c}\text { En mélange } \\
\text { Taux protéique } \\
\text { du régime } 20 \text { p. cent }\end{array}$ & Séparément \\
\hline 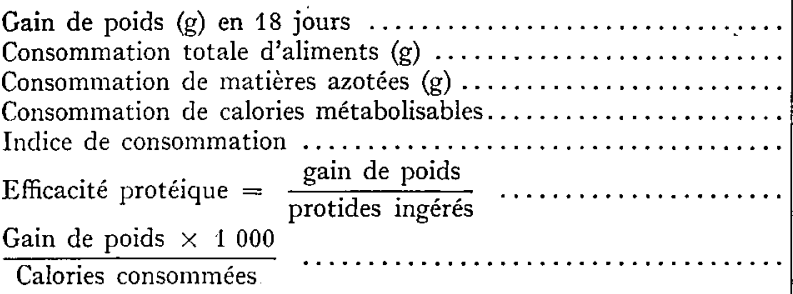 & $\begin{array}{r}216,0 \\
461,0 \\
92,3 \\
1620,0 \\
2,13 \\
2,37\end{array}$ & $\begin{array}{r}251,4 \\
551,0 \\
92,0 \\
1818,0 \\
2,18 \\
2,72\end{array}$ \\
\hline
\end{tabular}

Lorsque le poussin ingère la même quantité de matière azotée, la libre consommation de substances énergétiques s'accompagne d'une amélioration non négligeable de la croissance. Cette amélioration ne se manifeste pas au niveau de l'indice de consommation mais est la conséquence d'une consommation plus élevée de calories. II en résulte d'ailleurs que le gain de poids par calorie ingérée n'est pas modifié par le mode de rationnement. L'élévation de 1'ingestion d'énergie se traduit au niveau de l'efficacité azotée qui est augmentée lorsque les substances énergétiques et azotées du régime sont distribuées séparément.

L'ensemble de ces résultats montrent que la nature des matières azotées du régime retentit considérablement sur la consommation spontanée d'énergie du poussin. Il en résulte que la valeur du rapport des calories aux matières azotées du régime dépend, elle aussi, de l'origine des protides alimentaires, comme cela est figuré dans le tableau 3 .

TABLEAU 3

Variation du rapport Calories/Matières Azotées selon la nature des protides alimentaires.

\begin{tabular}{|c|c|c|c|}
\hline . & Protides ingérés (g) & $\begin{array}{l}\text { Consommation spontanée } \\
\text { de calories métabolisables }\end{array}$ & $\frac{\begin{array}{c}\text { Rapport } \\
\text { Calories métabolisables }\end{array}}{\text { Matières azotées }}$ \\
\hline $\begin{array}{l}\text { Arachide } \ldots \ldots \ldots \ldots \ldots \\
\text { Poisson } \ldots \ldots \ldots \ldots \ldots\end{array}$ & $\begin{array}{l}92,4 \\
92,0\end{array}$ & $\begin{array}{l}1420 \\
1818\end{array}$ & $\begin{array}{l}15,56 \\
21,32\end{array}$ \\
\hline
\end{tabular}

Pour une même quantité de protide ingéré, la consommation spontanée d'énergie augmente de $30 \mathrm{p}$. Ioo lorsque l'on remplace le tourteau d'arachide par la farine de poisson. Ia valeur biologique de la protéine retentit sur la valeur optimale du rapport Calories/Matières Azotées. 


\section{DISCUSSION.}

Quelle que soit la méthode de rationnement, la diversité des valeurs biologiques de l'arachide et du poisson apparaît clairement. Toutefois, la divergence s'accuse lorsque le poussin est en mesure de consommer la quantité de calories qui lui convient. Alors que pour un protide peu efficace, le système d'alimentation n'a pas de répercussion sur le gain de poids de l'animal, la consommation en libre choix des substances énergétiques non azotés permet une meilleure croissance au poussin nourri d'un protide de plus haute valeur. Il apparaît avec une grande netteté que la consommation spontanée d'énergie varie considérablement avec la nature de la source azotée.

Pour la plupart des auteurs, il existe un rapport optimum entre les calories et le pourcentage de protéines du régime. Lorsque le poussin est âgé de 3 semaines et reçoit une ration dosant $20 \mathrm{p}$. Ioo de matières azotées, le régime doit renfermer 17,5 calories métabolisables par gramme de protéine. Toutefois cette valeur optimale est sujette à de nombreux facteurs de variations dont les principaux sont : l'âge de l'animal, les taux protéique et énergétique du régime, 1'origine des calories.

On n'a jamais mis en évidence d'une manière claire le rôle des différentes matières azotées sur la valeur de ce rapport. LEONG, SUNDE, BIRD et ELVEHJEM, I959, constatent que le poussin manifeste une meilleure tolérance du régime hyperénergétique torsque les protéines sont mieux équilibrées. De même CoLEOU, I959, observe qu'il faut majorer les normes classiquement admises, lorsque le poussin reçoit des quantités importantes de lait écrémé dans son régime.

Toutes ces études ont été poursuivies en utilisant un régime complet où protéines et énergie sont distribuées en mélange. Lorsque l'on opère ainsi, on impose à l'animal qui ingère une quantité d'azote donnée, une ingestion d'une quantité proportionnelle de calories.

Cette manière d'agir ne semble pas satisfaisante pour permettre aux protides du régime de manifester leur pleine efficacité. La fourniture des principes énergétique et azoté dans un même rapport, sous forme de mélange (I7,5 calories métabolisables par gramme de matière azotée), se traduit dans nos essais par un excès de calories lorsque le régime contient de l'arachide, et par défaut lorsqu'il s'agit de la farine de poisson.

Ces premiers résultats semblent montrer que le poussin est capable de contrôler le niveau de son ingéré calorique en fonction de la qualité de la protéine alimentaire. Cette notion remettrait en question la signification des méthodes biologiques d'estimation de l'efficacité protéique.

Reçu en octobre 1960.

\section{SUMMARY}

VARIATION IN THE SPONTANEOUS CONSUMPTION OF ENERGY IN THE CHICK IN RELATION TO NITROGEN SOURCE OF THE DIET

The growth response of chickens receiving proteins of very different biological value has been studied with two different feeding systems. The proteins used were pea-nut or fish proteins given in limited and equal quantities, either separately or mixed with the diet.

In the first case, each bird was fed $25,5 \mathrm{~g}$ daily of complete diet containing $20 \mathrm{p}$. Ioo protein. 
In the second case, the bird was given simultaneous access to two feeders, one containing the nitrogen source (5, I $g$ protein per bird and per day), the other containing the nitrogen-free ration supplied ad libitum. The latter food intake is mesured daily in order to evaluate the spontaneous energy consumption of the chick according to the nature of the dietary protein.

The results show that an increase of $30 \mathrm{p}$. roo in the energy consumption is obtained when fish-meal is substituted for pea-nut oil-meal.

The following observations were made :

- Free choice consumption of calories fails to increase growth, compared to the other feeding system, in the case of pea-nut oil-meal ; but in the case of fish-meal, it leads to an improvement of weight gains.

- When the proteins are given mixed with the diet, their efficiency is not complete. Too many calories are given in the pea-nut diet, too little in the fish diet.

- The optimum calorie/protein ratio varies with the nature of the dietary protein.

\section{RÉFÉRENCES BIBLIOGRAPHIQUES}

BROBECK J. R., 1948. Food intake as mechanism of temperature regulation. Yale J. Biol. Med., 20, $545-55^{2}$.

Coleov, $\dot{J}$., r959, cité par Ferrando R., et Coleou J., I960. Problèmes essentiels et aspects nouveaux de l'alimentation du poulet de chair. Bull. Techn. Inform., n⿳⿲㇒丨丶 I 52, 491-499.

HARPER A. E., KUMTA U.S., I959. Amino acid balance and protein requirement. Fed. Proc., 18, I136-

II HILL F. W., DANSKY L. M., I954. Studies on the energy requirements of chickens. II. - The effect of dietary energy level on growth and feed consumption. Poult. Sci., 33, II2-IIg.

LEONG K. C., Sunde M. L., Bird H. R., ELVEhJEm C. A., I959. Interrelationships among dietary energy, protein, amino acids for chickens. Poult. Sci., 38, I 267-I285.

LEVEILLE G. A., FISHER H., 1958. The amino acid requirements for maintenance in the adult rooster. I. - Nitrogen and energy requirements in normal and protein-depleted animals receiving whole egg protein and amino acid diets. J. Nutr., 66, 44I-453.

Mac KITTRICK D. S., I947. The selection of chicks for growth experiments and the evaluation of growth. Growth, 11, 89-99.

MaYer J., Bates M. W., Van Itallie T. B., 1952. Blood sugar and food intake in rats with lesions of anterior hypothalamus. Metabolism, 1, 340-348.

Passmore R., RITCHIE F. J., r957. The specific dynamic action of food and the satiety mechanism. Brit. J. Nutr., 11, 79-85. 